\title{
THE SUSCEPTIBLE-INFECTIOUS MODEL OF DISEASE EXPANSION ANALYZED UNDER THE SCOPE OF CONNECTIVITY AND NEIGHBOR RULES
}

\author{
Maria Teresa Signes Pont, Higinio Mora Mora and Antonio Cortés \\ Castillo
}

Departamento de Tecnología Informática y Computación, Universidad de Alicante, Spain

teresa@dtic.ua.es; hmora@dtic.ua.es;

antoniocortescastillo@gmail.com

\begin{abstract}
This paper presents a model to approach the dynamics of infectious diseases expansion. Our model aims to establish a link between traditional simulation of the Susceptible-Infectious (SI) model of disease expansion based on ordinary differential equations (ODE), and a very simple approach based on both connectivity between people and elementary binary rules that define the result of these contacts. The SI deterministic compartmental model has been analysed and successfully modelled by our method, in the case of 4-connected neighbourhood.
\end{abstract}

\begin{abstract}
KEYWORDS
Infectious disease expansion, deterministic compartmental models, ODE, Neighbour binary rules, Connectivity.
\end{abstract}

\section{INTRODUCTION}

The outbreaks of infectious disease pandemics have shaped nations and civilizations through the ages. History tells us the terrible impact of biblical plagues in Ancient Egypt, bubonic plague in Europe in the Middle Age, influenza at the beginning of the twentieth century or AIDS the more recently emerging pandemics [1]. Mathematical modeling is playing a very important role to assess and control the potential outbreaks [2]. The first paper presenting a model for an infectious disease appears in 1760. The author, Bernouilli, a swiss mathematician and physicist, dealt with a statistical problem involving censored data in order to analyze smallpox morbidity and mortality that aimed to demonstrate the efficacy of vaccination [3]. At the beginning of the twentieth century two pioneering works can be mentioned. W.H. Hamer [4] published a discrete time epidemic model for the transmission of measles in 1906. The model assumes that the number of cases per unit time (incidence) depends on the product of densities of the susceptibles and infectives. In 1911 R. Ross [5] demonstrated that malaria is produced by the bite of a mosquito.

David C. Wyld et al. (Eds) : CSITA, ISPR, ARIN, DMAP, CCSIT, AISC, SIPP, PDCTA, SOEN - 2017

pp. 01- 10, 2017. (C) CS \& IT-CSCP 2017

DOI : 10.5121/csit.2017.70101 
Computer Science \& Information Technology (CS \& IT)

His mathematical model of expansion is based on a set of equations to approximate the discretetime dynamics of malaria and asserts is possible to control the disease whenever the population of mosquitos is reduced below a threshold. This was a new and crucial idea. Between 1927 and 1939 Kermack and McKendrick [6,7] published papers on epidemic models and obtained the epidemic threshold that the density of susceptibles must exceed for an epidemic outbreak to occur. This model includes three states, the $\mathrm{S}$ (susceptible), I (Infectious) and $\mathrm{R}$ (Recovered) instead of the two, S and I, of the Bernouilli's model. From the mid-twentieth century a great variety of epidemiological models have been developed after the recognition of the importance of modeling in public health decision making [8]. In the nineties, when the scientists began to pay attention to complex systems new paradigms spread out in order to better understand and model the impact of numerous variables that go beyond the micro host-pathogen level, such as ecological, social, economic, and demographic factors. Many scientists coming from such different fields as medicine, molecular biology, computer science and applied mathematics or economy have teamed up for rapid assessment of urgent situations of contagious diseases by means of a multidisciplinary approach. The case of HIV/AIDS pandemic [9-12] is a good example.

This paper presents a model to approach the dynamics of infectious diseases expansion by means of a set of neighbour rules between elements located in a lattice that represents the whole population. Following the introduction, Section 2 provides a brief summary of the deterministic compartmental models and highlights the Susceptible-Infectious (SI) model which has traditionally been solved by ODE. Section 3 presents our model which considers the population confined in a lattice. The contacts between people are performed by neighbour binary rules, that are tailored to model different situations such as Susceptible, Infected, with or without capability to infect further. The neighborhood is also defined depending of connectivity. We consider 4connection, 8-connection and horse jumping chess connection. The results are compared with those of the simulation of ODE. Section 4 presents a discussion upon the suitability of the model and proposes futures research. Section 5 summarises the work and presents concluding remarks.

\section{MATHEMATICAL MODELLING}

Three are the main categories encompassing mathematical modeling [1]. The statistical methods deal with real epidemics. They identify their spatial patterns and allow surveillance of outbreaks. The empirical models are based on machine learning methods such as data mining that allow the forecasting of the evolution of an ongoing epidemic spread. The mathematical or state-space methods provide quantitative predictions that have to be validated to forecast the evolution of a hypothetical or real epidemic spread. These methods also redefine our understanding of underlying mechanisms.

\subsection{The Deterministic Compartmental Models}

The description used in epidemiologic compartmental models is composed of standard categories represented by the variables that model the main characteristics of the system. These compartments, in the simplest case, divide the population into two health states: susceptible to the infection (denoted by S) and infected (denoted by I) [13]. The way that these compartments interact is often based upon phenomenological assumptions, and the model is built up from there. Usually these models are depicted by ODE, which are deterministic, but can also be viewed in more realistic stochastic framework [14]. To achieve more realism, other compartments are often included, namely the recovered (or removed or immune) compartment labelled by $\mathrm{R}$, or the 
exposed compartment, labelled by E. The stratification of these compartments lead to well-known models such as SIS [15], SIR [16], SEIR [17, 18],...or more complex ones [19]. The number of variables to be incorporated to the model depends on the particular disease being studied as well as on the desired complexity of the model. Other variables incorporated into the equation represent fundamental quantities such as birth rate, rate of transmission of infectious agent, death rate, and so forth, and are constants that can be changed.

\subsection{The Traditional Susceptible-Infectious (SI) Model}

In the SI model the two groups are the susceptible hosts, S, that are not infected by the pathogen but can get infected, and the infected hosts, I, who are infected by the pathogen. Assuming the mass-action model, the rate at which susceptible hosts become infected is a product of the number of contacts each host has per unit time, $r$, the probability of transmission of infection per contact, $\beta$, and the proportion of the host population that is infectious, $\mathrm{I} / \mathrm{N}$, where $\mathrm{N}=\mathrm{S}+\mathrm{I}$ is the total population size. This model is suitable to represent the case of the human immune deficiency virus (HIV) where there is no recovery. A schematic of the model is shown in Figure 1.

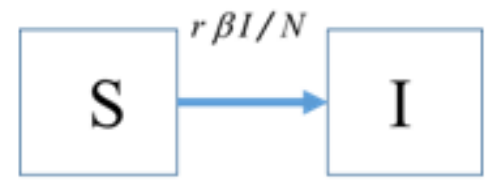

Figure 1. SI Model

Equations (1) for the SI model are as follows:

$$
\begin{aligned}
& \frac{d S}{d t}=-\beta r S \frac{I}{N} \\
& \frac{d I}{d t}=\beta r S \frac{I}{N}
\end{aligned}
$$

Since the population size is fixed, we can reduce the system to one dimension with the substitution $\mathrm{S}=\mathrm{N}-\mathrm{I}$ to provide the logistic Equation (2).

$$
\frac{d I}{d t}=\beta r(N-I) \frac{I}{N}
$$

We can analytically solve Equation (2) with the initial condition $\mathrm{I}(0)=\mathrm{I}_{0}$, so

$$
I(t)=\frac{I_{0} N}{\left(N-I_{0}\right) e^{-\beta r t}+I_{0}}
$$

The simulation of the SI model is shown in Figure 2 with the initial value $\mathrm{I}(0)=\mathrm{I}_{0}=1$ 


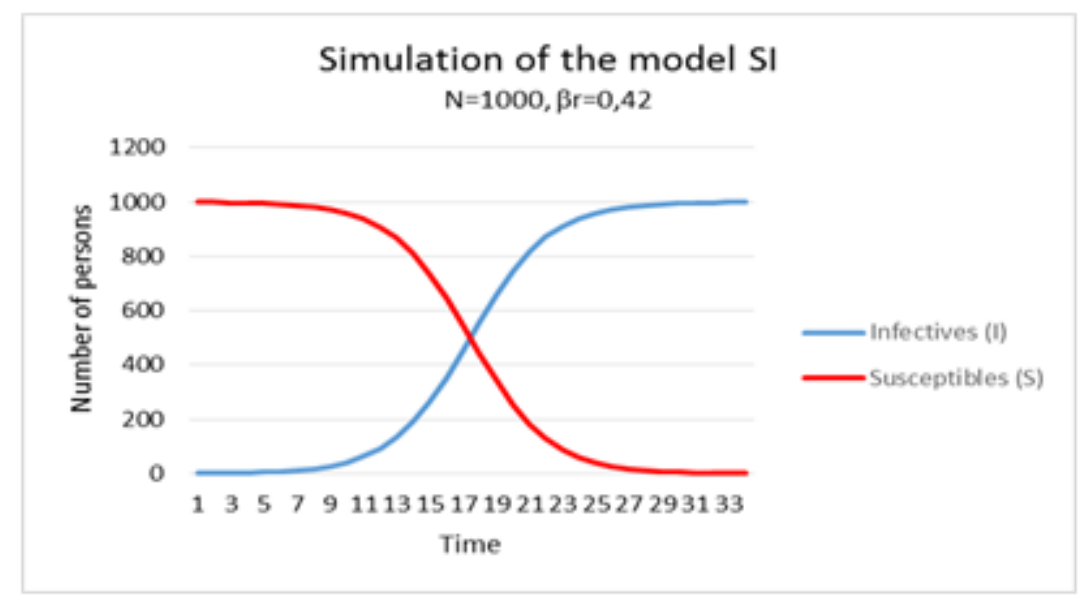

Figure 2. Simulation of the traditional SI Model

\section{OUR PROPOSAL}

Our proposal is based on a set of elementary binary rules that have the capability to model interactions between two individuals [20-23]. Without loss of generality we consider a twodimensional square lattice, every cell represents a susceptible person except the one at the center which locates an infected one. When the infected person contacts with his/her neighbors he/she spreads the disease. The new infected people have then the capability to infect other people.

\subsection{Binary neighbor rules}

Equation (4) defines a generic binary neighbor rule denoted $\otimes$.

$$
\begin{aligned}
& \otimes:(0,1) \rightarrow(0,1) \\
& (x, y) \rightarrow z=x \otimes y
\end{aligned}
$$

The $\otimes$ rule can be represented by a two input table that defines concretely the operation, as shown in Figure 3.

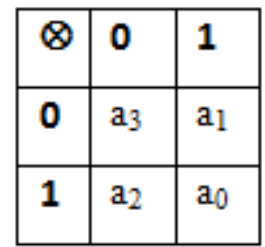

Figure 3. Generic neighbor rule represented by a table

Let $\mathrm{m}$ stand for the number of the rule. The number is represented by the four bits stored in the cells; $m=a_{3} a_{2} a_{1} a_{0}, \in\left[0,2^{4}-1\right] ; a_{i} \in(0,1) ; i \in[0,3]$. As an example, we consider $m=7$, that is to say $a_{3}=0 ; a_{2}=1 ; a_{1}=1$ and $a_{0}=1$. 


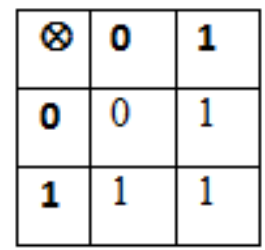

Figure 4 . The rule $n^{\circ} 7$ represented by a table $\left(a_{3}=0 ; a_{2}=1 ; a_{1}=1 ; a_{0}=1\right)$

The table defines concretely the operation as follows:

$0 \otimes 0=0 ; 0 \otimes 1=1 ; 1 \otimes 0=1$ and $1 \otimes 1=1$

This operation is suitable to model the interaction between infected people and susceptible people by identifying " 0 " as susceptible and " 1 " as infected. The previous operation means that infected people can transmit the disease to susceptible people $(1 \otimes 0=1)$, when infected people contact other infected people, all them remain infected $(1 \otimes 1=1)$, susceptible people have no effects upon people $(0 \otimes 0=0$ and $0 \otimes 1=1)$.

\subsection{Neighborhood rules modelling the spreading of a disease}

In the following two-dimensional square lattices we present the spreading of a disease by a unique Infected (" 1 ") located at the center of the lattice. All the empty cells are considered to be Susceptible ("0"). The red numbers stand for the generation number (time unit) the spreading occurs. Figure 5 shows the case of a $5 \times 5$ lattice with a contagion rate $\rho=4$ per generation (4connected cells are neighbors to every cell that touches one of their edges, following the Von Neumann neighbourhood).

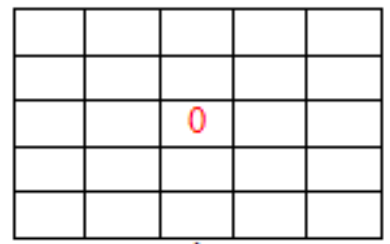

Generation0

\begin{tabular}{|l|l|l|l|l|}
\hline & 3 & 2 & 3 & \\
\hline 3 & 2 & 1 & 2 & 3 \\
\hline 2 & 1 & 0 & 1 & 2 \\
\hline 3 & 2 & 1 & 2 & 3 \\
\hline & 3 & 2 & 3 & \\
\hline
\end{tabular}

Generation 3

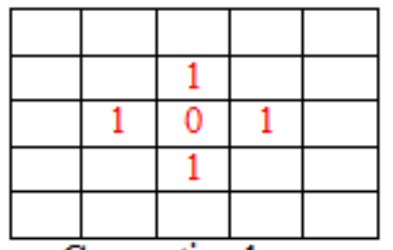

Generation 1

\begin{tabular}{|l|l|l|l|l|}
\hline 4 & 3 & 2 & 3 & 4 \\
\hline 3 & 2 & 1 & 2 & 3 \\
\hline 2 & 1 & 0 & 1 & 2 \\
\hline 3 & 2 & 1 & 2 & 3 \\
\hline 4 & 3 & 2 & 3 & 4 \\
\hline \multicolumn{6}{|c|}{ Geration 4}
\end{tabular}

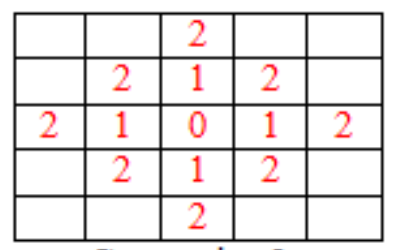

Generation2

\begin{tabular}{|c|c|c|c|}
\hline & S & I & New infections \\
\hline Gen.0 & 24 & 1 & 1 \\
\hline Gen.1 & 20 & 5 & 4 \\
\hline Gen.2 & 12 & 13 & 8 \\
\hline Gen.3 & 4 & 21 & 8 \\
\hline Gen.4 & 0 & 25 & 4 \\
\hline
\end{tabular}

Figure 5. The spreading of a disease in a 5x5 lattice with $\rho=4$ (4-connected cells)

The spreading of the disease results in a diamond-shaped region shown for rate $=4$ in Figure 5 . The evolution of the infected people can be carried out by means of the equation $1+2 \rho(\rho+1)$, where $\rho$ stands for the rate. 
The same example is presented for 8-connected cells (with horizontal, vertical, and diagonal connection) following the Moore neighborhood, and for the jumping chess neighbourhood. See Figures 6 and 7 respectively.

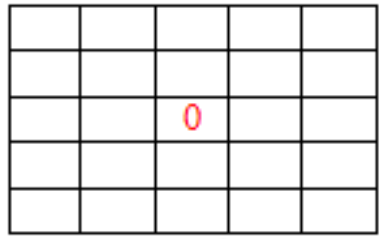

Generation0

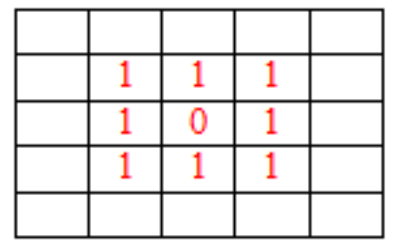

Generation 1

\begin{tabular}{|l|l|l|l|l|}
\hline 2 & 2 & 2 & 2 & 2 \\
\hline 2 & 1 & 1 & 1 & 2 \\
\hline 2 & 1 & 0 & 1 & 2 \\
\hline 2 & 1 & 1 & 1 & 2 \\
\hline 2 & 2 & 2 & 2 & 2 \\
\hline
\end{tabular}

Generation2

\begin{tabular}{|c|c|c|c|}
\hline & S & I & $\begin{array}{c}\text { New } \\
\text { Infections }\end{array}$ \\
\hline Gen.0 & 24 & 1 & 1 \\
\hline Gen.1 & 16 & 9 & 8 \\
\hline Gen.2 & 0 & 25 & 16 \\
\hline
\end{tabular}

Figure 6 . The spreading of a disease in a $5 \times 5$ lattice with $\rho=8$ (8-connected cells)

For the 8-connected neighborhood, the evolution of the infected people can be carried out by means of the equation $(2 p+1)^{2}$.
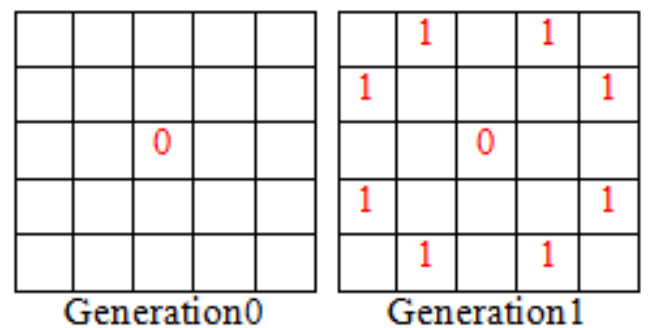

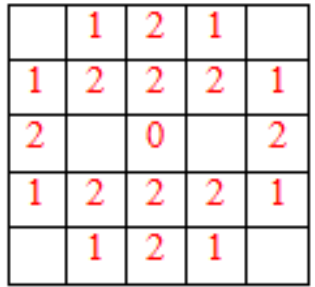

Generation2

\begin{tabular}{|l|l|l|l|l|}
\hline 3 & 1 & 2 & 1 & 3 \\
\hline 1 & 2 & 2 & 2 & 1 \\
\hline 2 & 3 & 0 & 3 & 2 \\
\hline 1 & 2 & 2 & 2 & 1 \\
\hline 3 & 1 & 2 & 1 & 3 \\
\hline \multicolumn{5}{|c|}{ Gen mation 3 }
\end{tabular}

Generation3

\begin{tabular}{|c|c|c|c|}
\hline & S & I & $\begin{array}{c}\text { New } \\
\text { Infections }\end{array}$ \\
\hline Gen.0 & 24 & 1 & 1 \\
\hline Gen.1 & 16 & 9 & 8 \\
\hline Gen.2 & 6 & 19 & 10 \\
\hline Gen. 3 & 0 & 25 & 6 \\
\hline
\end{tabular}

Figure 7. The spreading of a disease in a $5 \times 5$ lattice with $\rho=8$ (horse jumping chess connected cells)

\subsection{Comparison Between the Traditional SI Model and the 4-Connected Neigborhood Model}

The following graph represents the 4-connected case in a 32x32 lattice, equivalent to $\mathrm{N}=1024$ (in order to approximate the graphic shown in Figure 2 where $\mathrm{N}=1000$ ). 


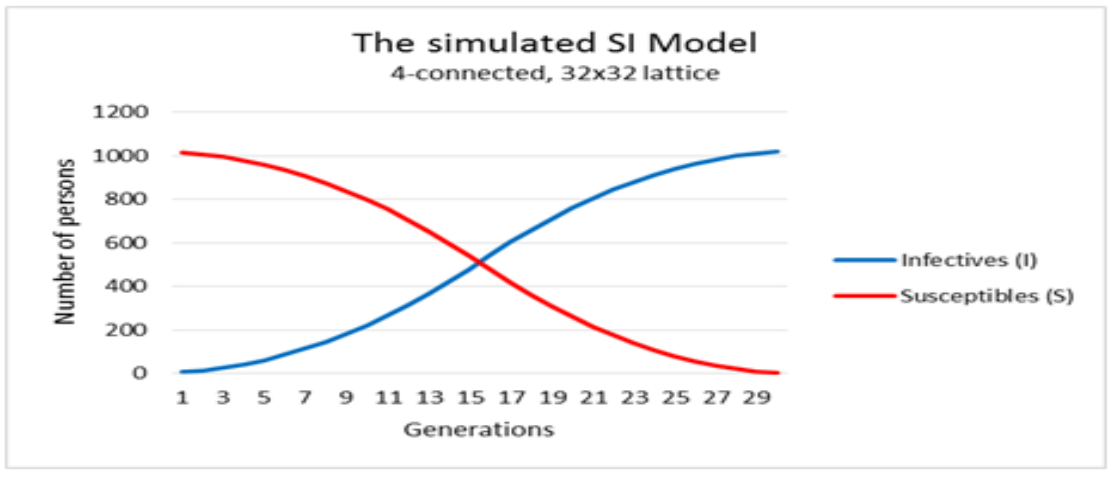

Figure 8 . The spreading of a disease in a $32 \times 32=1024$ lattice with $\rho=4$ (4 -connected cells).

The comparison between Figures 2 and 8 suggests that the 4-connected cells (Von Neumann neighbourhood) could be a suitable approximation to approach the traditional SI model. In order to better compare the models, we now compare the previous simulation based on Equation 2 with our 4-connected neighborhood model for similar populations, lattices $10 x 10$, equivalent to $\mathrm{N}=100$ and 100x100, equivalent to $\mathrm{N}=10000$, See Figures 9 and 10.
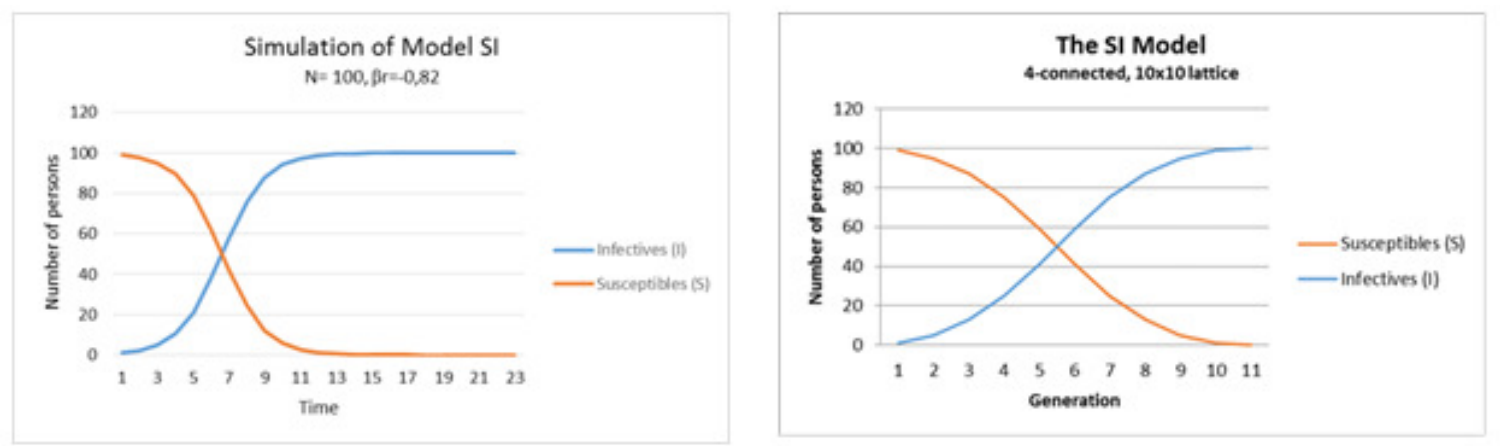

Figure 9. The spreading of a disease in a $10 \times 10$ lattice with a $\rho=4$ (4 -connected cells), compared to the simulation of the traditional model $(\mathrm{N}=100, \beta \mathrm{r}=0,82)$.
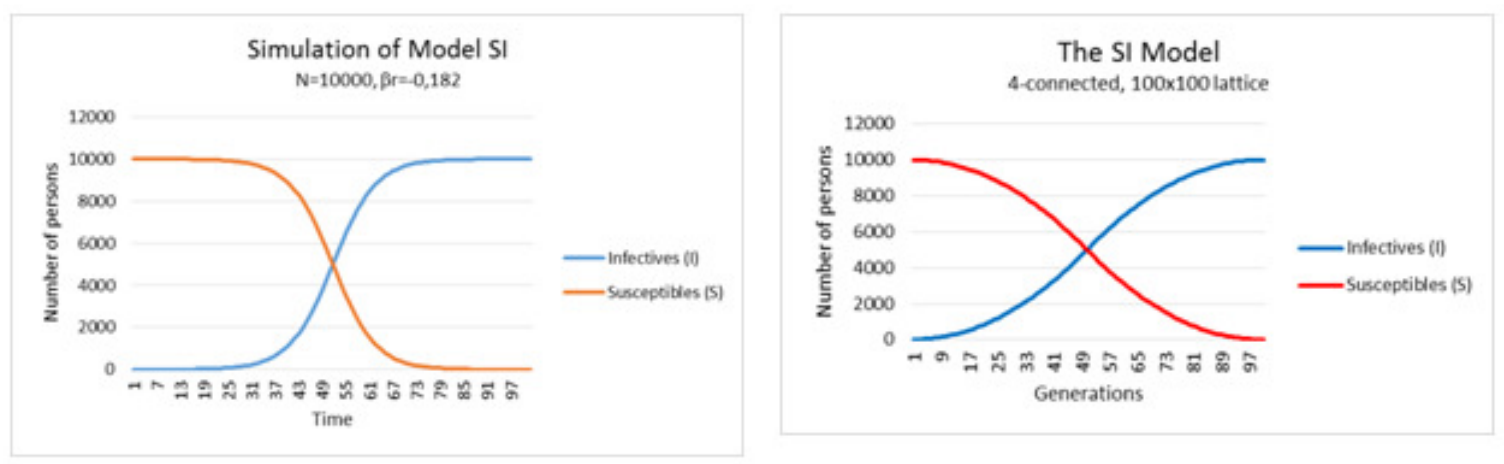

Figure 10. The spreading of a disease in a $100 \times 100$ lattice with $\rho=4$ (4 -connected cells), compared to the simulation of the traditional model $(\mathrm{N}=10000, \beta \mathrm{r}=0,182)$. 
Computer Science \& Information Technology (CS \& IT)

Empirically, the rate $\beta \mathrm{r}$ of the traditional simulation of Model SI has been modified when $\mathrm{N}$ varies in order to impose the crossing point between (I) and (S) occurs at the same "time" (obviously we have assumed that "Time" in the traditional model is equivalent to "Generation" in ours, as explained in Section 4.).

\section{DISCUSSION}

Our model establishes a link between the traditional ODE simulation of the SI deterministic compartmental model of disease expansion and a very simple model based on both the connectivity between people and different rules that define the results of the contacts. The parameters of each model must harmonize. Our lattice size stands for $\mathrm{N}$, the number of persons $(\mathrm{N}=\mathrm{S}+\mathrm{I})$. In the ODE model, the number of contacts each host has per unit time, $r$, and the probability of transmission of infection per contact, $\beta$, have been englobed in a unique variable, $\beta r$, which stands for the number of actual infections that occur in a unit time, which is equivalent to the connectivity, $\rho$, in our model. Obviously, the time scale is different in each model, so, unit time may be days, hours, etc... In our model, "Generation" is a dimensionless unit, which only means how the sequence of infections occur. In order to allow meaningful comparisons between the different approaches we have harmonized the parameters of the models as follows, $\mathrm{N} \equiv$ size of the lattice and $\beta r \equiv \rho$. In this initial paper we have equalized the values of the studied population $(\mathrm{N}=100$ or 10000 vs lattice size $10 \times 10$ or $100 \times 100$, respectively) as well as the crossing point of the plotted values of $S$ and I, which is 49 , by tuning the value of $\beta r$ (see Figures 9 and 10). This empirical approach reveals the capability of our model to meet the desired values. For a more refined modelling some attention should be paid to the slopes, by means of a balanced choice of (lattice size, rate, generation).

\section{CONCLUSION}

We have presented a new approach to the SI deterministic compartmental model. Our proposal is based on both connectivity between people and elementary binary rules that quantify the contacts between people. Our model fits the results carried out by traditional simulation of ODE. This encouraging empirical result must be improved in the future by means of a deeper analysis of the connectivity including a probabilistic approach of it. The SIR, SIS, SIRS models will be also studied. Finally, experimental data coming from statistics on real cases must be directly confronted in order to validate our model. Further we envisage to adapt this model to the field of virus expansion in computers.

\section{REFERENCES}

[1] Siettos, C.I. \& Russo, L. Mathematical modeling of infectious disease dynamics Virulence (2013) May 15; 4(4): 295-306.

[2] Choisy, M. et al. Mathematical Modeling of Infectious Diseases Dynamics. In Encyclopedia of Infectious Diseases: Modern Methodologies, by M.Tibayrenc Copyright (C) 2007 John Wiley \& Sons,Inc.

[3] Bernoulli, D. (Reprinted in Blower, S. 2004). An attempt at a new analysis of the mortality caused by smallpox and of the advantages of inoculation to prevent it. Reviews in medical virology, 14 (5): 275-88. 
[4] Hamer, W.H. Epidemic Disease in England: The Evidence of Variability and of Persistency of Type. Bedford Press, 1906.

[5] Ross, R. The Prevention of Malaria. Murray, London, 1911.

[6] Kermack, W.O \& McKendrick, A.G. A contribution to the mathematical theory of epidemics, Proc. R. Soc. Lond. 115 (1927), 700-721.

[7] Isea, R \& Lonngren, K.E. On the Mathematical Interpretation of Epidemics by Kermack and McKendrick. Gen. Math. Notes, Vol. 19, No. 2, December 2013, pp. 83-87.

[8] Bailey NJT. The Mathematical Theory of Infectious Diseases and its Application. Griffin, London, 1957.

[9] Culshaw, R.V. Mathematical Modeling of AIDS Progression: Limitations, Expectations, and Future Directions. Ph.D. Journal of American Physicians and Surgeons, Vol 11, No4, Winter 2006.

[10] Marshall, Brandon D. L. et al. A Complex Systems Approach to Evaluate HIV Prevention in Metropolitan Areas: Preliminary Implications for Combination Intervention Strategies. PLOS ONE, Vol.7, (9), 2012.

[11] Naresh, R. et al. Modelling and analysis of the spread of AIDS epidemic with immigration of HIV infectives. Mathematical and Computer Modelling, Vol. 49, Issues 5-6, March 2009, pp. 880-892.

[12] Cassels, S. \& and Goodreau, S.M. Interaction of mathematical modeling and social and behavioral HIV/AIDS research. Curr Opin HIV AIDS. 2011 Mar; 6(2): 119-123.

[13] Van den Driessche, P. Detreministic Compartmental Models: Extension of Basic Models. Lecture Notes in Mathematics, Mathematical Epidemiology, Springer, 2008.

[14] Gillespie, D.T. (1977). Exact Stochastic Simulation of Coupled Chemical Reactions". The Journal of Physical Chemistry, 81 (25), pp. 2340-2361.

[15] Ricard, M.R. et al. Epidemiological model with fast dispersion. Proceedings of BIOMAT 2005, R. P. Mondaini \& R. Dilao Ed.

[16] Ahmed A \& M. Hassan. A New Solution of SIR Model by using the Differential Fractional Transformation method. International Journal of Engineering and Applied Science, April, 2014. Vol. 4, No. 11

[17] Legrand, J. et al. Understanding the dynamics of Ebola epidemics. Epid. Infect. May 2007; 135(4) $610-621$.

[18] http://www.math.washington.edu/ morrow/mcm/mcm15/38725paper.pdf

[19] Iseal, R. \& Lonngren, K.E. A Preliminary Mathematical Model for the Dynamic Transmission of Dengue, Chikungunya and Zika. American Journal of Modern Physics and Application 2016; 3(2): $11-15$

[20] Signes Pont, M.T. et al. A Computational Approach of the French Flag Model to Connect Growth and Specification in Developmental Biology, Cognitive Computation, September 2016. 
[21] Signes Pont, M.T. et al. Computational primitive to model the emergence of behavioral patterns. Proceedings of the 3rd WSEAS International Conference on Automatic control, Soft Computing and Human-machine Interaction in Recent Advances in Electrical Engineering Series 49; 2015, pp.80104.

[22] Signes Pont, M.T. et al. An approach to stigmergy issues based on the recursive applications of binary elementary operations. Proceedings of World Conference on Information Systems and Technologies. In Advances in Intelligent Systems and Computing, Vol. 275, 2014, pp.191-197.

[23] Signes Pont, M.T. et al. An approach to computations in living tissues based on logic functions. Proceedings of 20th Euromicro Conference on Parallel, Distributed and Network-Based Processing, 2012.

\section{AUTHORS}

Maria Teresa Signes Pont received the BS degree in Computer Science at the Institut National des Sciences Appliquées, Toulouse (France) and the BS degree in Physics at the Universidad Nacional de Educación a Distancia (Spain). She received the $\mathrm{PhD}$ in Computer Science at the University of Alicante in 2005. Since 1996, she is a member of the Computer Science and Technology department of the same university where she is currently an associate professor and researcher. Her areas of research at the Specialized Processors Architecture Laboratory include computer arithmetic and the design of floating point units, approximation algorithms related to VLSI design and natural systems modelling.

Higinio Mora Mora received the BS degree in computer science engineering and the BS degree in business studies in University of Alicante, Spain, in 1996 and 1997, respectively. He received the $\mathrm{PhD}$ degree in computer science from the University of Alicante in 2003. Since 2002, he is a member of the faculty of the Computer Technology and Computation Department at the same university where he is currently an associate professor and researcher of Specialized Processors Architecture Laboratory. His areas of research interest include computer modelling, computer architectures, high performance computing, embedded systems, internet of things and cloud computing paradigm.

Antonio Cortes Castillo is a computer engineer trained in the Latin American University of Science and Technology (ULACIT), Costa Rica, 1995. He obtained his bachelor's degree in computer engineering with an emphasis in Management Information Systems at the National University of Heredia, Costa Rica, 2002. Adquiere his Master's degree in Computer Science (Telematics) at the Technological Institute of Costa Rica. He is now teaching at the University of Panama and is with his $\mathrm{PhD}$. at the University of Alicante.
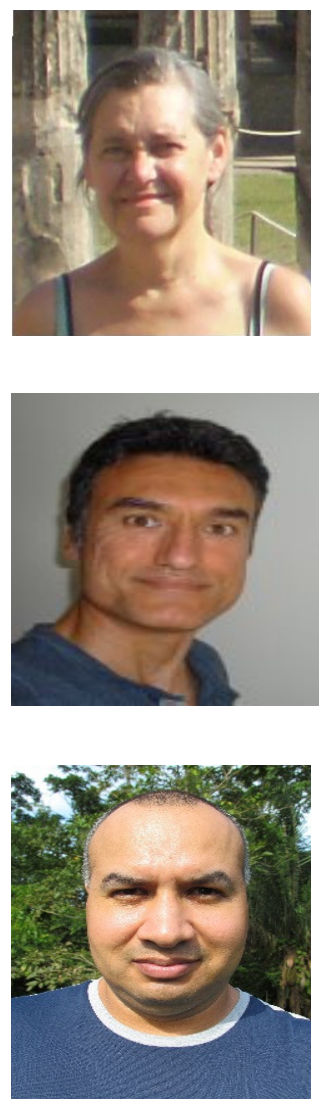